\title{
Front Matter: Volume 7739
}

, "Front Matter: Volume 7739," Proc. SPIE 7739, Modern Technologies in Space- and Ground-based Telescopes and Instrumentation, 773901 (16 August 2010); doi: 10.1117/12.873435

SDIE Event: SPIE Astronomical Telescopes + Instrumentation, 2010, San Diego, SPIE. California, United States 


\title{
PROCEEDINGS OF SPIE
}

\section{Modern Technologies in Space- and Ground-based Telescopes and Instrumentation}

\author{
Eli Atad-Ettedgui \\ Dietrich Lemke \\ Editors
}

27 June-2 July 2010

San Diego, California, United States

Sponsored by

SPIE

Cooperating Organizations

American Astronomical Society (United States) - Association of Universities for Research in Astronomy, Inc. (United States) - Astronomical Society of Japan (Japan) - Atacama Large Millimeter/submillimeter Array • Ball Aerospace \& Technologies Corporation (United States) Canadian Astronomical Society (CASCA) (Canada) • Commissariat à l'Energie Atomique (France) • European Astronomical Society (Switzerland) • ESO-European Organisation for Astronomical Research in the Southern Hemisphere (Germany) • Japan Aerospace Exploration Agency (Japan) • Jet Propulsion Laboratory (United States) • NASA Goddard Space Flight Center (United States) • National Astronomical Observatory Japan (Japan) National Radio Astronomy Observatory - SOFIA-Stratospheric Observatory for Infrared Astronomy (United States) - Thirty Meter Telescope Project (United States) • W. M. Keck Observatory (United States)

Published by

SPIE

Volume 7739 
The papers included in this volume were part of the technical conference cited on the cover and title page. Papers were selected and subject to review by the editors and conference program committee. Some conference presentations may not be available for publication. The papers published in these proceedings reflect the work and thoughts of the authors and are published herein as submitted. The publisher is not responsible for the validity of the information or for any outcomes resulting from reliance thereon.

Please use the following format to cite material from this book:

Author(s), "Title of Paper," in Modern Technologies in Space- and Ground-based Telescopes and Instrumentation, edited by Eli Atad-Ettedgui, Dietrich Lemke, Proceedings of SPIE Vol. 7739 (SPIE, Bellingham, WA, 2010) Article CID Number.

ISSN 0277-786X

ISBN 9780819482297

Published by

SPIE

P.O. Box 10, Bellingham, Washington $98227-0010$ USA

Telephone +1 3606763290 (Pacific Time) · Fax +1 3606471445

SPIE.org

Copyright (C) 2010, Society of Photo-Optical Instrumentation Engineers

Copying of material in this book for internal or personal use, or for the internal or personal use of specific clients, beyond the fair use provisions granted by the U.S. Copyright Law is authorized by SPIE subject to payment of copying fees. The Transactional Reporting Service base fee for this volume is $\$ 18.00$ per article (or portion thereof), which should be paid directly to the Copyright Clearance Center (CCC), 222 Rosewood Drive, Danvers, MA 01923. Payment may also be made electronically through CCC Online at copyright.com. Other copying for republication, resale, advertising or promotion, or any form of systematic or multiple reproduction of any material in this book is prohibited except with permission in writing from the publisher. The CCC fee code is 0277-786X/10/\$18.00.

Printed in the United States of America.

Publication of record for individual papers is online in the SPIE Digital Library.

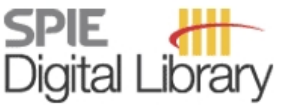

SPIEDigitalLibrary.org

Paper Numbering: Proceedings of SPIE follow an e-First publication model, with papers published first online and then in print and on CD-ROM. Papers are published as they are submitted and meet publication criteria. A unique, consistent, permanent citation identifier (CID) number is assigned to each article at the time of the first publication. Utilization of CIDs allows articles to be fully citable as soon they are published online, and connects the same identifier to all online, print, and electronic versions of the publication. SPIE uses a six-digit CID article numbering system in which:

- The first four digits correspond to the SPIE volume number.

- The last two digits indicate publication order within the volume using a Base 36 numbering system employing both numerals and letters. These two-number sets start with 00, 01, 02, 03, 04 , 05, 06, 07, 08, 09, OA, OB ... 0Z, followed by 10-1Z, 20-2Z, etc.

The CID number appears on each page of the manuscript. The complete citation is used on the first page, and an abbreviated version on subsequent pages. Numbers in the index correspond to the last two digits of the six-digit CID number. 


\title{
Contents
}

\section{Part One}

\author{
xxiii Conference Committee \\ xxv Introduction \\ xxvii Unknowns and unknown unknowns: from dark sky to dark matter and dark energy \\ (Plenary Paper) [7733-501] \\ Y. Suto, The Univ. of Tokyo (Japan) \\ xxxix Optical synoptic telescopes: new science frontiers (Plenary Paper) [7733-502] \\ J. A. Tyson, Univ. of California, Davis (United States)
}

\section{SESSION 1 OPTICS FABRICATION I}

773902 Measurement of aspheric mirror segments using Fizeau interferometry with CGH correction (Invited Paper) [7739-32]

J. H. Burge, C. Zhao, M. Dubin, College of Optical Sciences, The Univ. of Arizona (United States)

773903 Fabrication technologies for large optical components at Carl Zeiss Jena GmbH [7739-02] G. Derst, V. Giggel, Carl Zeiss Jena GmbH (Germany)

773905 Precision grinding for rapid fabrication of segments for extremely large telescopes using the Cranfield BoX [7739-04]

X. Tonnellier, P. Morantz, P. Shore, P. Comley, Cranfield Univ. (United Kingdom)

$773907 \quad M 1$ and $\mathbf{M} 2$ mirror manufacturing for ARIES project: current status [7739-06]

A. P. Semenov, M. A. Abdulkadyrov, A. P. Patrikeev, V. E. Patrikeev, V. V. Pridnya, Joint Stock Co. Lytkarino Optical Glass Factory (Russian Federation)

$773908 \quad$ Ultra-precisely manufactured mirror assemblies with well-defined reference structures [7739-07]

S. Scheiding, Fraunhofer Institute for Applied Optics and Precision Engineering (Germany) and Friedrich-Schiller-Univ. Jena (Germany); C. Damm, Fraunhofer Institute for Applied Optics and Precision Engineering (Germany); W. Holota, Holota Optics (Germany); T. Peschel, A. Gebhardt, S. Risse, Fraunhofer Institute for Applied Optics and Precision Engineering (Germany); A. Tünnermann, Fraunhofer Institute for Applied Optics and Precision Engineering (Germany) and Friedrich-Schiller-Univ. Jena (Germany) 
7739 OA Fabrication and testing of the first 8.4-m off-axis segment for the Giant Magellan Telescope (Invited Paper) [7739-09]

H. M. Martin, R. G. Allen, Steward Observatory, The Univ. of Arizona (United States); J. H. Burge, Steward Observatory, The Univ. of Arizona (United States) and College of Optical Sciences, The Univ. of Arizona (United States); D. W. Kim, College of Optical Sciences, The Univ. of Arizona (United States); J. S. Kingsley, M. T. Tuell, S. C. West, Steward Observatory, The Univ. of Arizona (United States); C. Zhao, T. Zobrist, College of Optical Sciences, The Univ. of Arizona (United States)

7739 OB Narrow ion-beam figuring: a new tool to address extreme slopes on small surfaces located near telescope pupils [7739-10]

U. Mueller, J. Stone, B. G. Peters, J. Daniel, L-3 Communications Tinsley Labs. Inc. (United States); T. P. Greene, R. Belikov, NASA Ames Research Ctr. (United States); O. Guyon, Subaru Telescope, National Astronomical Observatory of Japan (United States)

7739 OC Lightweight high-performance 1-4 meter class spaceborne mirrors: emerging technology for demanding spaceborne requirements [7739-118]

T. Hull, L-3 Communications Tinsley Labs. Inc. (United States); P. Hartmann, SCHOTT AG

(Germany); A. R. Clarkson, L-3 Communications Brashear (United States); J. M. Barentine, L-3 Communications Tinsley Labs. Inc. (United States); R. Jedamzik, T. Westerhoff, SCHOTT AG (Germany)

$7739 \mathrm{OE}$ Investigation of sub-aperture polish techniques for manufacturing astronomical mirror [7739-107]

Y. Zheng, X. Li, W. Guo, Z. Dong, B. Liang, Z. Jiang, Nanjing Institute of Astronomical Optics \& Technology (China)

7739 OF On the super polishing under stress of aspherical surfaces for exoplanet detection and solar instruments [7739-13]

E. Hugot, M. Ferrari, S. Vives, M. Laslandes, K. El Hadi, S. Moindrot, G. R. Lemaitre, K. Dohlen, Lab. d'Astrophysique de Marseille, CNRS, Aix-Marseille Univ. (France)

7739 OG Low-weight, low-cost, low-cycle time, replicated glass mirrors [7739-14]

R. Egerman, S. De Smitt, D. Strafford, ITT Geospatial Systems (United States)

\section{SESSION 3 MATERIALS/METROLOGY}

$7739 \mathrm{OH} \quad$ Composite panels for optical mirrors for Cherenkov Telescopes: development of the cold glass slumping technology [7739-15]

R. Canestrari, INAF, Osservatorio Astronomico di Brera (Italy) and Univ. degli Studi dell'Insubria (Italy); G. Motta, INAF, Osservatorio Astronomico di Brera (Italy) and Univ. degli Studi di Milano-Bicocca (Italy); G. Pareschi, S. Basso, INAF, Osservatorio Astronomico di Brera (Italy); M. Doro, Istituto Nazionale di Fisica Nucleare (Italy); E. Giro, L. Lessio, INAF,

Osservatorio Astronomico di Padova (Italy)

7739 OI Modeling of the thermal expansion behaviour of ZERODUR at arbitrary temperature profiles [7739-16]

R. Jedamzik, T. Johansson, T. Westerhoff, SCHOTT AG (Germany) 
7739 0J Designing optimized ultra-lightweighted mirror structures made of Cesic for space and ground based applications [7739-17]

P. Hofbauer, M. R. Krödel, ECM Ingenieur-Unternehmen für Energie- und Umwelttechnik $\mathrm{GmbH}$ (Germany)

7739 OK Material characteristics of CLEARCERAM-Z HS for use in large diameter mirror blanks [7739-111]

T. Kishi, N. Goto, Ohara Inc. (Japan); B. Hoffman, Ohara Corp. (United States); Y. Kawashima, K. Suzuki, K. Yoshizawa, K. Suzuki, Ohara Inc. (Japan)

$7739 \mathrm{OL}$ The center of curvature optical assembly for the JWST primary mirror cryogenic optical test [7739-19]

C. Wells, G. Olczak, C. Merle, T. Dey, M. Waldman, T. Whitman, E. Wick, A. Peer, ITT

Geospatial Systems (United States)

7739 OM Manufacturing of the ZERODUR 1.5-m primary mirror for the solar telescope GREGOR as preparation of light weighting of blanks up to 4-m diameter [7739-20]

T. Westerhoff, M. Schäfer, A. Thomas, M. Weissenburger, T. Werner, A. Werz, SCHOTT AG (Germany)

\section{SESSION 4 TEST AND METROLOGY I}

$7739 \mathrm{ON}$ Alignment and use of the optical test for the 8.4-m off-axis primary mirrors of the Giant Magellan Telescope (Invited Paper) [7739-21]

S. C. West, Steward Observatory, The Univ. of Arizona (United States); J. H. Burge, College of Optical Sciences, The Univ. of Arizona (United States); B. Cuerden, W. Davison, J. Hagen, H. M. Martin, M. T. Tuell, Steward Observatory, The Univ. of Arizona (United States); C. Zhao, T. Zobrist, College of Optical Sciences, The Univ. of Arizona (United States)

773900 Automated metrology simulator for multi-objects instruments [7739-22] E. Atad-Ettedgui, C. Dickson, S. Beard, X. Gao, B. Stobie, P. Hastings, H. M. McGregor, UK Astronomy Technology Ctr. (United Kingdom)

$7739 \mathrm{OP}$ Interference testing methods of large astronomical mirrors base on lenses and CGH wavefront correctors [7739-23]

M. A. Abdulkadyrov, S. P. Belousov, V. E. Patrikeev, A. P. Semenov, Joint Stock Co. Lytkarino Optical Glass Factory (Russian Federation)

$77390 Q$ Saving SALT: repairs to the spherical aberration corrector of the Southern African Large Telescope (SALT) [7739-24]

D. E. O'Donoghue, J. O'Connor, L. A. Crause, F. Strumpfer, South African Astronomical Observatory (South Africa); O. J. Strydom, J. D. Brink, SALT Foundation (South Africa); C. Sass, South African Astronomical Observatory (South Africa); E. Wiid, SALT Foundation (South Africa); E. Atad-Ettedgui, UK Astronomy Technology Ctr. (United Kingdom)

7739 OR Metrology of complex astigmatic surfaces for astronomical optics [7739-25]

S. Rolt, A. K. Kirby, D. J. Robertson, Durham Univ. (United Kingdom) 
7739 OS Accuracy of laser tracker measurements of the GMT 8.4-m off-axis mirror segments [7739-26]

T. L. Zobrist, J. H. Burge, College of Optical Sciences, The Univ. of Arizona (United States) and Steward Observatory, The Univ. of Arizona (United States); H. M. Martin, Steward Observatory, The Univ. of Arizona (United States)

\section{SESSION 5 TEST AND METROLOGY II}

7739 OT Imaging issues for interferometry with CGH null correctors [7739-27]

J. H. Burge, C. Zhao, P. Zhou, College of Optical Sciences, The Univ. of Arizona (United States)

7739 OU Metrology systems for active alignment control of the Hobby-Eberly Telescope wide field corrector [7739-28]

H. Lee, G. J. Hill, McDonald Observatory, The Univ. of Texas at Austin (United States); M. Hart, Hart Scientific Consulting International L.L.C. (United States); M. E. Cornell, R. Savage, B. Vattiat, D. Perry, W. M. Moller, T. Rafferty, T. Taylor, M. D. Rafal, McDonald Observatory, The Univ. of Texas at Austin (United States)

7739 OV Optical testing of aspheres based on photochromic computer-generated holograms [7739-29]

G. Pariani, Politecnico di Milano (Italy) and Osservatorio Astronomico di Brera (Italy); A. Bianco, Osservatorio Astronomico di Brera (Italy); C. Bertarelli, Politecnico di Milano (Italy) and Ctr. for Nano Science and Technology (Italy); P. Spanó, E. Molinari, Osservatorio Astronomico di Brera (Italy)

7739 OX SALT segmented primary mirror: laboratory test results for FOGALE inductive edge sensors [7739-132]

J. Menzies, H. Gajjar, S. Buous, D. Buckley, P. Gillingham, South African Large Telescope, South African Astronomical Observatory (South Africa)

7739 OY Interferometric test method for testing convex aspheric mirror surfaces [7739-31]

T. S. McKechnie, McKechnie Optics Research (United States)

\section{SESSION $6 \quad$ TEST AND METROLOGY III}

773910 Liquid deformable mirror for advanced sub-optical system testing [7739-33]

S. Thibault, D. Brousseau, E. F. Borra, Univ. Laval (Canada)

773911 Scanning pentaprism test for the GMT 8.4-m off-axis segments [7739-34]

R. Allen, Steward Observatory, The Univ. of Arizona (United States); P. Su, College of Optical Sciences, The Univ. of Arizona (United States); J. H. Burge, Steward Observatory, The Univ. of Arizona (United States) and College of Optical Sciences, The Univ. of Arizona (United States); B. Cuerden, H. M. Martin, Steward Observatory, The Univ. of Arizona (United States)

773912 A novel optical sensor for mirror edge sensing [7739-128]

D. A. H. Buckley, S. Buous, H. Gajjar, J. W. Menzies, South African Astronomical Observatory (South Africa) and Southern African Large Telescope Observatory (South Africa);

F. Schindler, K. Sändig, Dr. Johannes Heidenhain GmbH (Germany); S. Lévêque, European Organisation for Astronomical Research in the Southern Hemisphere (Germany) 
773913 Development of a large mosaic volume phase holographic (VPH) grating for APOGEE [7739-36]

J. Arns, Kaiser Optical Systems, Inc. (United States); J. C. Wilson, M. Skrutskie, Univ. of Virginia (United States); S. Smee, R. Barkhouser, The Johns Hopkins Univ. (United States); D. Eisenstein, Steward Observatory, The Univ. of Arizona (United States); J. Gunn, Princeton Univ. (United States); F. Hearty, Univ. of Virginia (United States); A. Harding, The Johns Hopkins Univ. (United States); P. Maseman, Univ. of Virginia (United States); J. Holtzman, New Mexico State Univ. (United States); R. Schiavon, Gemini Observatory (United States); B. Gillespie, Apache Point Observatory (United States); S. Majewski, Univ. of Virginia (United States)

773914 The Vector Vortex Coronagraph: sensitivity to central obscuration, low-order aberrations, chromaticism, and polarization [7739-37]

D. Mawet, L. Pueyo, D. Moody, J. Krist, E. Serabyn, Jet Propulsion Lab. (United States)

\section{SESSION 7 CRYOGENIC SPACE INSTRUMENTS}

773915 The innovative MAIT plan for the Matisse cold optics, comprising an unprecedented 220 cryogenic optical components [7739-167]

E. Elswijk, R. Navarro, N. Tromp, J. Kragt, NOVA, ASTRON (Netherlands); J. Pragt, NOVA, ASTRON (Netherlands) and ASTRON (Netherlands); H. Hanenburg, A. van Duin, ASTRON (Netherlands)

773916 Thermal architecture of the Clover cryostats [7739-165]

W. F. Grainger, P. A. R. Ade, P. Hargrave, Cardiff Univ. (United Kingdom); S. Chase, Chase Research Cryogenics Ltd. (United Kingdom); B. Kiernan, C. E. North, Cardiff Univ. (United Kingdom)

773917 Flight model performance of the integral field unit for the James Webb Space Telescope's near-infrared spectrograph [7739-40]

D. J. Purll, D. R. Lobb, A. R. Barnes, Surrey Satellite Technology Ltd. (United Kingdom);

R. G. Talbot, S. Rolt, D. J. Robertson, Durham Univ. (United Kingdom); M. F. Closs, EADS Astrium GmbH (Germany); M. te Plate, European Space Agency (Netherlands)

773918 High-precision cryogenic wheel mechanisms of the JWST/MIRI instrument: performance of the flight models [7739-41]

O. Krause, F. Müller, S. Birkmann, A. Böhm, M. Ebert, U. Grözinger, Th. Henning, R. Hofferbert, A. Huber, D. Lemke, R.-R. Rohloff, S. Scheithaver, Max-Planck-Institut für Astronomie (Germany); T. Gross, T. Fischer, G. Luichtel, H. Merkle, M. Übele, H.-U. Wieland, Carl Zeiss Optronics GmbH (Germany); J. Amiaux, CEA, IRFU (France); R. Jager, ASTRON (Netherlands); A. Glauser, ETH Zurich (Switzerland); P. Parr-Burman, UK Astronomy Technology Ctr. (United Kingdom); J. Sykes, Univ. of Leicester (United Kingdom)

773919 Accurate blackbodies [7739-42]

H. M. Latvakoski, M. Watson, S. Topham, D. Scott, M. Wojcik, G. Bingham, Space Dynamics Lab., Utah State Univ. (United States)

7739 1A A filter wheel mechanism for the Euclid near-infrared imaging photometer [7739-43]

R. Holmes, U. Grözinger, O. Krause, Max-Planck-Institut für Astronomie (Germany);

M. Schweitzer, Max-Planck-Institut für extraterrestrische Physik (Germany) 
7739 1B Cryogenic magnetic bearing scanning mechanism design for the SPICA/SAFARI Fourier transform spectrometer [7739-44]

T. C. van den Dool, R. F. M. M. Hamelinck, B. Kruizinga, W. L. M. Gielesen, B. C. Braam,

J. R. Nijenhuis, TNO S\&l (Netherlands); N. Loix, S. Luyckx, Micromega Dynamics SA (Belgium);

D. van Loon, P. P. Kooijman, SRON Nationaal Instituut voor Ruimteonderzoek (Netherlands);

B. M. Swinyard, Space Science and Technology Dept., Rutherford Appleton Lab. (United

Kingdom)

SESSION 8

TELESCOPE STRUCTURE I

7739 1C A control loop closure system for the Sardinia Radio Telescope active surface [7739-46] C. Pernechele, C. Barbieri, P. Bolli, F. Buffa, T. Pisanu, S. Poppi, G. Serra, INAF, Osservatorio Astronomico di Cagliari (Italy); M. Morsiani, J. Roda, G. Zacchiroli, C. Nocita, M. Paternò, INAF, Istituto di Radioastronomia (Italy)

7739 1D A prototype micro-autonomous positioning system for mirror deployment within multi-object instruments [7739-48]

W. D. Taylor, Institute for Astronomy (United Kingdom); D. C. Atkinson, W. A. Cochrane, D. M. Montgomery, H. Schnetler, T. E. C. Baillie, UK Astronomy Technology Ctr. (United Kingdom); S. Clausen, Heriot-Watt Univ. (United Kingdom)

7739 IE Starbugs: focal plane fiber positioning technology [7739-49]

M. Goodwin, J. Heijmans, I. Saunders, J. Brzeski, W. Saunders, R. Muller, R. Haynes, J. Gilbert, Anglo-Australian Observatory (Australia)

7739 IF Vibration damping system for ALMA antenna transporters [7739-50]

M. Dimmler, M. Kraus, L. Kern, N. Di Lieto, European Southern Observatory (Germany)

7739 1G Study on a novel panel support concept for radio telescopes with active surface [7739-52] D. Yang, G. Zhou, Nanjing Institute of Astronomical Optics \& Technology (China); D. Okoh, Univ. of Nigeria (Nigeria); G. Li, Nanjing Institute of Astronomical Optics \& Technology (China); J. Cheng, National Radio Astronomy Observatory (United States)

773911 GREGOR M1 mirror and cell design: effects of different mirror substrates on the telescope design [7739-54]

M. Süß, MT Mechatronics GmbH (Germany); R. Volkmer, Kiepenhever-Institut für Sonnenphysik (Germany); P. Eisenträger, MT Mechatronics GmbH (Germany)

7739 IJ LSST telescope primary/tertiary mirror hardpoints [7739-142]

J. DeVries, D. Neill, E. Hileman, National Optical Astronomy Observatory (United States)

7739 IK Friction drive characterization breadboard: test results [7739-51]

B. Sedghi, C. Lucuix, European Southern Observatory (Germany); J. M. Tortolani, AMOS Ltd. (Belgium); E. Brunetto, European Southern Observatory (Germany); C. Delrez, E. Gabriel, AMOS Ltd. (Belgium)

7739 1L Innovative enclosure design for the MROI array telescopes [7739-144]

I. Payne, New Mexico Institute of Mining and Technology, Magdalena Ridge Observatory

(United States); G. Marchiori, A. Busatta, European Industrial Engineering s.r.l. (Italy) 
7739 1M Smart structures for deformable mirrors actuated by shape memory alloy [7739-56] M. Riva, INAF, Osservatorio Astronomico di Brera (Italy); P. Bettini, L. Di Landro, G. Sala, Politecnico di Milano (Italy); F. M. Zerbi, INAF, Osservatorio Astronomico di Brera (Italy)

7739 IN Smart structures for deformable mirrors actuated by piezocomposites (Invited Paper) [7739-57]

M. Riva, INAF, Osservatorio Astronomico di Brera (Italy); D. Di Sanzo, A. Airoldi, G. Sala, Politecnico di Milano (Italy); F. M. Zerbi, INAF, Osservatorio Astronomico di Brera (Italy)

773910 EST Telescope: primary mirror, support, and cooling system [7739-58]

R. Volkmer, Kiepenhever-Institut für Sonnenphysik (Germany); F. Manni, M. Giannuzzi, A. Scotto, S.R.S. Engineering Design s.r.I. (Italy); L. Cavaller, GRANTECAN S.A. (Spain); T. Scheiffelen, Kiepenheuer-Institut für Sonnenphysik (Germany); F. Bettonvil, Utrecht Univ. (Netherlands); F. Berrilli, Univ. degli Studi di Roma Tor Vergata (Italy)

7739 IP Calibration dome screen for the Large Synoptic Survey Telescope [7739-59]

W. J. Gressler, National Optical Astronomy Observatory (United States); P. Doherty, Harvard Univ. (United States); V. L. Krabbendam, M. Liang, A. Saha, National Optical Astronomy Observatory (United States); C. W. Stubbs, A. Vaz, Harvard Univ. (United States)

$77391 Q \quad$ New paradigms for producing high-performing meter class ground-based telescopes [7739-60]

R. L. Hedrick, A. Keller, J. Haberman, K. lott, PlaneWave Instruments (United States); T. B. Hull, L-3 Communications Tinsley Labs. Inc. (United States); A. R. Clarkson, L-3 Brashear (United States); D. Rowe, Sierra Monolithics, Inc. (United States); D. Gray, Sidereal Technology (United States); R. Genet, Consultant

7739 IR High-precision robotic equatorial C-ring telescope mounts: design, fabrication, and performance [7739-61]

M. A. Dubberley, Las Cumbres Observatory Global Telescope Network (United States)

7739 is Design and performance characterization of the LCOGTN One-Meter Telescope optical tube assembly (Invited Paper) [7739-62]

B. J. Haldeman, R. M. Haynes, V. Posner, J. R. Tufts, A. J. Pickles, M. A. Dubberley, Las Cumbres Observatory Global Telescope Network (United States)

7739 IT The active optics system for the Discovery Channel Telescope [7739-63]

B. Smith, T. Chylek, Lowell Observatory (United States); B. Cuerden, College of Optical Sciences, The Univ. of Arizona (United States); B. DeGroff, P. J. Lotz, A. Venetiou, Lowell Observatory (United States)

7739 iU Challenges of extreme load hexapod design and modularization for large ground-based telescopes [7739-64]

R. Gloess, Physik Instrumente GmbH \& Co. KG (Germany); B. Lula, PI (Physik Instrumente) L.P. (United States) 
$77391 \mathrm{~V}$ Innovative enclosure dome/observing aperture system design for the MROI Array Telescopes [7739-65]

A. Busatta, G. Marchiori, S. Mian, European Industrial Engineering s.r.l. (Italy); I. Payne, New Mexico Institute of Mining and Technology, Magdalena Ridge Observatory (United States); M. Pozzobon, European Industrial Engineering s.r.l. (Italy)

\section{SESSION 10 COATINGS}

7739 IW Are opthalmic hydrophobic coatings useful for astronomical optics? [7739-66] C. Schwab, Univ. of Heidelberg (Germany); A. C. Phillips, Univ. of California Observatories (United States)

7739 1X Design and manufacturing of high-performance notch filters [7739-67]

U. Schallenberg, B. Ploss, M. Lappschies, S. Jakobs, Optics Balzers Jena GmbH (Germany)

7739 IY The achromatic chessboard, a new concept of a phase shifter for nulling interferometry III: experimental performances [7739-68]

D. Pickel, LESIA, Observatoire de Paris, CNRS, UPMC, Univ. Paris Diderot (France) and LUTH, Observatoire de Paris, CNRS, Univ. Paris Diderot (France); D. Rouan, LESIA, Observatoire de Paris, CNRS, UPMC, Univ. Paris Diderot (France); D. Pelat, LUTH, Observatoire de Paris, CNRS, Univ. Paris Diderot (France); J.-M. Reess, O. Dupuis, LESIA, Observatoire de Paris, CNRS, UPMC, Univ. Paris Diderot (France); F. Chemla, M. Cohen, GEPI, Observatoire de Paris, CNRS, Univ. Paris Diderot (France)

773912 Experimental advances in phase mask coronagraphy [7739-69] M. N'Diaye, Lab. d'Astrophysique de Marseille, CNRS, Univ. de Provence (France) and Instituto de Astronomía, Univ. Nacional Autónoma de México (Mexico); K. Dohlen, Lab. d'Astrophysique de Marseille, CNRS, Univ. de Provence (France); S. Cuevas, Instituto de Astronomía, Univ. Nacional Autónoma de México (Mexico); R. Soummer, Space Telescope Science Institute (United States); C. Sánchez, Univ. Nacional Autónoma de México (Mexico)

773920 Verification of the controllability of refractive index by subwavelength structure fabricated by photolithography: toward single-material mid- and far-infrared multilayer filters [7739-70] H. Makitsubo, The Univ. of Tokyo (Japan) and Japan Aerospace Exploration Agency (Japan); T. Wada, M. Mita, Japan Aerospace Exploration Agency (Japan)

773921 Assessment of black and spectrally selective surfaces for stray light reduction in telescope systems [7739-71]

S. M. Pompea, National Optical Astronomy Observatory (United States)

\section{SESSION 11 PHOTONICS}

773922 The OPTICON technology roadmap for optical and infrared astronomy (Invited Paper) [7739-72]

C. Cunningham, D. Melotte, UK Astronomy Technology Ctr. (United Kingdom); F. Molster, NOVA (Netherlands) 
773923 Direct laser written multimode waveguides for astronomical applications [7739-73] N. Jovanovic, Macquarie Univ. (Australia) and Australian Astronomical Observatory (Australia); S. Gross, C. Miese, A. Fuerbach, Macquarie Univ. (Australia) and Ctr. for Ultrahigh bandwidth Devices for Optical Systems (Australia); J. Lawrence, Macquarie Univ. (Australia) and Australian Astronomical Observatory (Australia); M. J. Withford, Macquarie Univ. (Australia) and Ctr. for Ultrahigh bandwidth Devices for Optical Systems (Australia)

773924 Characterising modal noise in fibre-coupled spectrographs for astronomy [7739-194] U. Lemke, J. Corbett, J. Allington-Smith, G. Murray, Durham Univ. (United Kingdom)

773925 Defining requirements and identifying relevant technologies in astrophotonics [7739-75] J. R. Allington-Smith, Durham Univ. (United Kingdom); T. A. Birks, Univ. of Bath (United Kingdom); J. Bland-Hawthorn, The Univ. of Sydney (Australia) and Anglo-Australian Observatory (Australia); C. R. Cunningham, UK Astronomy Technology Ctr. (United Kingdom); S. Dagupta, Univ. of Southampton (United Kingdom); R. Haynes, Anglo-Australian Observatory (Australia), Astrophysikalisches Institut Potsdam (Germany), and InnoFSPEC (Germany); P. J. V. Garcia, Univ. do Porto (Portugal); A. K. Kar, Heriot-Watt Univ. (United Kingdom); A. Kelz, Astrophysikalisches Institut Potsdam (Germany) and InnoFSPEC (Germany); P. Y. Kern, Lab. d'Astrophysique de l'Observatoire de Grenoble (France); L. Labadie, Instituto de Astrofísica de Canarias (Spain); J. S. Lawrence, Macquarie Univ. (Australia); E. P. Le Coarer, Lab. d'Astrophysique de l'Observatoire de Grenoble (France); M. M. Roth, Astrophysikalisches Institut Potsdam (Germany) and InnoFSPEC (Germany); S. Minardi, Friedrich-Schiller-Univ. Jena (Germany); R. M. Sharples, Durham Univ. (United Kingdom); R. R. Thomson, Heriot-Watt Univ. (United Kingdom)

773926 Supercontinuum light sources for use in astronomical instrumentation: a test with PMAS, the Potsdam multi-aperture spectrophotometer [7739-76]

M. M. Roth, Astrophysikalisches Institut Potsdam (Germany) and innoFSPEC Potsdam (Germany); H.-G. Löhmannsröben, Univ. Potsdam (Germany) and innoFSPEC Potsdam (Germany); C. Dosche, Univ. Potsdam (Germany); C. Sandin, Astrophysikalisches Institut Potsdam (Germany) and innoFSPEC Potsdam (Germany); O. Reich, Univ. Potsdam (Germany) and innoFSPEC Potsdam (Germany); R. Haynes, Astrophysikalisches Institut Potsdam (Germany) and innoFSPEC Potsdam (Germany); L. Leick, NKT Photonics A/S (Denmark); J. M. Chávez Boggio, Astrophysikalisches Institut Połsdam (Germany) and innoFSPEC Potsdam (Germany); A. Kelz, Astrophysikalisches Institut Potsdam (Germany)

773927 Optical design of optical switches for diverse field spectroscopy [7739-177]

R. Content, G. J. Murray, J. R. Allington-Smith, Durham Univ. (United Kingdom)

773928 Dither-based sensor for improved consistency of adaptive optics system [7739-78]

A. Vyas, Indian Institute of Astrophysics (India) and Indian Institute of Science (India);

M. B. Roopashree, B. R. Prasad, Indian Institute of Astrophysics (India) 


\section{Part Two}

773929 Ground-based observatory operations optimized and enhanced by direct atmospheric measurements [7739-79]

J. T. McGraw, P. C. Zimmer, M. R. Ackermann, D. C. Hines, A. B. Hull, L. Rossmann,

D. C. Zirzow, The Univ. of New Mexico (United States); S. W. Brown, G. T. Fraser, K. R. Lykke, A. W. Smith, National Institute of Standards and Technology (United States); C. W. Stubbs, Harvard niv. (United States); J. T. Woodward, National Institute of Standards and Technology (United States)

$77392 \mathrm{~B}$ Fibre positioning revisited: the use of an off-the-shelf assembly robot for OPTIMOS-EVE [7739-192]

G. B. Dalton, Rutherford Appleton Lab. (United Kingdom) and Univ. of Oxford (United Kingdom); M. S. Whalley, Rutherford Appleton Lab. (United Kingdom); O. Mounissamy, Univ. of Oxford (United Kingdom) and IUT Paris Jussieu, Univ. Paris Diderot (France); E. C. Sawyer, I. A. J. Tosh, D. L. Terrett, Rutherford Appleton Lab. (United Kingdom); I. J. Lewis, Univ. of Oxford (United Kingdom)

$77392 \mathrm{C} \quad$ Fibre Bragg gratings for temporal spectral astronomy [7739-81]

G. Mariën, N. Cvetojevic, Macquarie Univ. (Australia); N. Jovanovic, Macquarie Univ. (Australia) and Australian Astronomical Observatory (Australia); J. Dawes, Macquarie Univ. (Australia); J. Bland-Hawthorn, Sydney Institute for Astronomy, Univ. of Sydney (Australia); R. Haynes, innoFSPEC, Astrophysikalisches Institut Potsdam (Germany); J. Lawrence, Q. Parker, Macquarie Univ. (Australia) and Australian Astronomical Observatory (Australia); M. J. Withford, Macquarie Univ. (Australia)

\section{POSTER SESSION: OPTICS FABRICATION/MATERIALS}

7739 2D Fabrication of 4-meter class astronomical optics [7739-104] M. J. Valente, D. W. Kim, C. J. Oh, M. J. Novak, J. H. Burge, College of Optical Sciences, The Univ. of Arizona (United States)

$77392 \mathrm{E}$ Studies on evaluating and removing subsurface damage on the ground surface of CLEARCERAM-Z HS [7739-105]

H. Akitaya, T. Yamashita, N. Ohshima, M. Iye, National Astronomical Observatory of Japan (Japan); T. Maihara, H. Tokoro, K. Takahashi, Nano-Optonics Research Institute (Japan)

$77392 \mathrm{G}$ Carbon fiber reinforced composites: their structural and thermal properties [7739-108] J. Cheng, National Radio Astronomy Observatory (United States); D. Yang, Nanjing Institute of Astronomical Optics \& Technology (China)

$77392 \mathrm{H} \quad$ Structural analysis of a new type lightweight optical mirror blank [7739-109]

Y. Li, X. Cui, National Astronomical Observatories, Nanjing Institute of Astronomical Optics \& Technology (China) and Key Lab. of Astronomical Optics \& Technology, Nanjing Institute of Astronomical Optics \& Technology (China); N. Hu, The Civilizer New Products Institute Nanjing (China) 
773921 Herschel Space Telescope: impact of new material strain data on optical test and model correlation [7739-1 12]

B. Catanzaro, CFE Services (United States); D. Doyle, European Space Research and Technology Ctr. (Netherlands); E. Cohen, CFE Services (United States)

7739 2J Post-flight reflectance of COSTAR and WF/PC 2 pickoff mirrors upon their return from space [7739-113]

M. A. Quijada, R. M. Henry, T. Madison, R. Boucarut, J. G. Hagopian, NASA Goddard Space Flight Ctr. (United States)

7739 2K Ultra-lightweighted HB-Cesic one-meter mirror demonstrator [7739-114]

M. R. Krödel, P. Hofbaver, ECM Ingenieur-Unternehmen für Energie- und Umwelttechnik GmbH (Germany)

7739 2L Recent achievements with a cryogenic ultra-lightweighted HB-Cesic mirror [7739-115] M. R. Krödel, P. Hofbaver, ECM Ingenieur-Unternehmen für Energie- und Umwelttechnik GmbH (Germany); C. Devilliers, Thales Alenia Space (France); Z. Sodnik, European Space Research and Technology Ctr. (Netherlands); P. Robert, Société Européenne de Systèmes Optiques (France)

7739 2M CFRP composite mirrors for space telescopes and their micro-dimensional stability [7739-116]

S. Utsunomiya, T. Kamiya, R. Shimizu, Japan Aerospace Exploration Agency (Japan)

$77392 \mathrm{~N}$ Time-dependent deformation of surface geometry on light weight and thermally stable CFRP mirror in humid environment [7739-117]

Y. Arao, Waseda Univ. (Japan); J. Koyanagi, S. Utsunomiya, S. Takeda, Japan Aerospace Exploration Agency (Japan); H. Kawada, Waseda Univ. (Japan)

773920 Secondary mirror system for the European Solar Telescope (EST) [7739-1 19]

L. Cavaller, B. Siegel, G. Prieto, GRANTECAN S.A. (Spain); E. Hernandez, Instituto de

Astrofísica de Canarias (Spain); J. M. Casalta, J. Mercader, J. Barriga, NTE-SENER S.A. (Spain)

\section{POSTER SESSION: TEST AND METROLOGY}

$77392 P \quad$ An improved method of the spatial point's position detection [7739-120] Y. Zhang, X. Li, Univ. of Science and Technology of China (China); L. Zhu, National Astronomical Observatories (China); J. Jin, W. Li, Univ. of Science and Technology of China (China)

$77392 \mathrm{~A}$ A method of attitude measurement of the calibration target [7739-121]

J. Jin, X. Li, Univ. of Science and Technology of China (China); L. Zhu, National Astronomical Observatories (China); Y. Zhang, B. Li, W. Li, Univ. of Science and Technology of China (China)

7739 2R A method of 3D reconstruction based on single camera [7739-122]

L. Zhu, National Astronomical Observatories (China); X. Li, B. Li, J. Jin, Y. Zhang, W. Li, Univ. of Science and Technology of China (China) 
773925 Use of a Faro Arm for optical alignment [7739-123]

L. A. Crause, D. E. O'Donoghue, J. E. O'Connor, F. Strümpfer, South African Astronomical Observatory (South Africa)

7739 2T Surface measurements of radio antenna panels with white-light interferometry [7739-124] S. Chinellato, Univ. degli Studi di Padova (Italy); C. Pernechele, INAF, Astronomical Observatory of Cagliari (Italy); S. Carmignato, Univ. degli Studi di Padova (Italy); F. Manzan, INAF, Astronomical Observatory of Cagliari (Italy)

$77392 \mathrm{~V}$ Optical testing of the LSST combined primary/tertiary mirror [7739-126] M. T. Tuell, H. M. Martin, Steward Observatory, The Univ. of Arizona (United States); J. H. Burge, Steward Observatory, The Univ. of Arizona (United States) and College of Optical Sciences, The Univ. of Arizona (United States); W. J. Gressler, National Optical Astronomy Observatory (United States); C. Zhao, College of Optical Sciences, The Univ. of Arizona (United States)

7739 2W Advanced wavefront sensing and control testbed (AWCT) [7739-130] F. Shi, S. A. Basinger, R. T. Diaz, R. O. Gappinger, H. Tang, R. K. Lam, E. Sidick, R. C. Hein, M. Rud, M. Troy, Jet Propulsion Lab. (United States)

$77392 X \quad$ Phase retrieval methods for wavefront sensing [7739-131]

S. Bikkannavar, D. Redding, J. Green, S. Basinger, D. Cohen, J. Lou, C. Ohara, F. Shi, Jet Propulsion Lab. (United States)

$77392 \mathrm{Y}$ First cophasing of a segmented mirror with a tunable filter and the pyramid wavefront sensor [7739-135]

M. Bonaglia, E. Pinna, A. Puglisi, S. Esposito, Osservatorio Astrofisico di Arcetri (Italy);

J. C. Guerra, Isaac Newton Group of Telescopes (Spain); R. Myers, N. Dipper, Durham Univ. (United Kingdom)

\section{POSTER SESSION: TELESCOPE STRUCTURE AND MECHANICAL DESIGN}

773930 An innovative low-cost antenna dish built with commercial off-the-shelf (COTS) components [7739-136]

J. Cheng, J. Ruff, S. Sturgis, National Radio Astronomy Observatory (United States); D. Yang, Nanjing Institute of Astronomical Optics \& Technology (China)

773931 The VST active primary mirror support system [7739-137]

P. Schipani, INAF, Osservatorio Astronomico di Capodimonte (Italy); M. Capaccioli, S. D'Orsi, L. Ferragina, L. Marty, C. Molfese, INAF, VSTCeN (Italy); F. Perrotta, INAF, Osservatorio Astronomico di Capodimonte (Italy); G. De Paris, D. Fierro, INAF, Sede Centrale (Italy); R. Tomelleri, P. Rossettini, F. Perina, S. Recchia, Tomelleri s.r.l. (Italy); D. Magrin, INAF, Osservatorio Astronomico di Padova (Italy)

773932 Performance of the VST secondary mirror support system [7739-138]

P. Schipani, INAF, Osservatorio Astronomico di Capodimonte (Italy); S. D'Orsi, D. Fierro, L. Marty, INAF, VSTCeN (Italy); F. Perrotta, INAF, Osservatorio Astronomico di Capodimonte (Italy); C. Arcidiacono, INAF, Osservatorio Astrofisico di Arcetri (Italy) 
773933 The axial actuators for the VST primary mirror [7739-139]

D. Fierro, INAF (Italy); S. D'Orsi, L. Marty, Tomelleri s.r.l. (Italy); C. Molfese, F. Perrotta, P. Schipani, INAF, Osservatorio Astronomico di Capodimonte (Italy); M. Capaccioli, Tomelleri s.r.I. (Italy); G. De Paris, INAF (Italy); R. Tomelleri, P. F. Rossettini, Tomelleri s.r.l. (Italy); J. Farinato, INAF, Osservatorio Astronomico di Padova (Italy)

773935 Thermal behavior of the Medicina 32-meter radio telescope [7739-141]

T. Pisanu, F. Buffa, National Institute for Astrophysics, Cagliari Astronomical Observatory (Italy); M. Morsiani, National Institute for Astrophysics, Institute of Radio Astronomy (Italy); C. Pernechele, S. Poppi, National Institute for Astrophysics, Cagliari Astronomical Observatory (Italy)

773936 Fast force actuators for LSST primary/tertiary mirror [7739-143] E. Hileman, National Optical Astronomy Observatory (United States); M. Warner, Cerro Tololo Inter-American Observatory (Chile); O. Wiecha, National Optical Astronomy Observatory (United States)

773937 Innovative relocation system for enclosures for MROI array telescopes [7739-145] A. Busatta, L. Ghedin, G. Marchiori, S. Mian, European Industrial Engineering s.r.l. (Italy); I. Payne, New Mexico Institute of Mining and Technology, Magdalena Ridge Observatory (United States); M. Pozzobon, European Industrial Engineering s.r.l. (Italy)

POSTER SESSION: SIMULATION, TESTING, AND CONTROLS

773938 Design and simulation of the direct drive servo system [7739-147]

C. Ren, Z. Liu, L. Song, Q. Yi, K. Chen, Tsinghua Univ. (China); Z. Zhang, Nanjing Institute of Astronomical Optics \& Technology (China)

773939 Toward high-dynamic active mirrors for LGS refocusing systems [7739-148]

E. Hugot, F. Madec, S. Vives, M. Ferrari, D. Le Mignant, J. G. Cuby, Lab. d'Astrophysique de Marseille, CNRS, Univ. de Provence (France)

7739 3A In-flight aberrations corrections for large space telescopes using active optics [7739-149] M. Laslandes, M. Ferrari, E. Hugot, G. Lemaitre, Lab. d'Astrophysique de Marseille, CNRS, Univ. de Provence (France)

7739 3B The calibration and evaluation for laser tracker application in LAMOST site environment [7739-151]

Z. Zhou, Y. Jin, C. Zhai, Y. Gu, Univ. of Science and Technology of China (China)

7739 3C Performance verification testing for HET wide-field upgrade tracker in the laboratory [7739-152]

J. Good, McDonald Observatory, The Univ. of Texas at Austin (United States); R. Hayes, J. Beno, The Univ. of Texas at Austin (United States); J. Booth, M. E. Cornell, G. J. Hill, H. Lee, McDonald Observatory, The Univ. of Texas at Austin (United States); J. Mock, The Univ. of Texas at Austin (United States); M. Rafal, R. Savage, McDonald Observatory, The Univ. of Texas at Austin (United States); I. Soukup, The Univ. of Texas at Austin (United States) 
7739 3D Upgrading the controller of the fast tip-tilt tertiary mirror for the SOAR Telescope [7739-153] M. Warner, Cerro Tololo Inter-American Observatory (Chile); S. Heathcote, SOAR Telescope (Chile); G. Schumacher, R. Cantarutti, E. Parkes, Cerro Tololo Inter-American Observatory (Chile)

\section{POSTER SESSION: CRYOGENICS, MECHANISMS, INSTRUMENTS}

7739 3E LN2 continuous flow cryostats: a compact vibration free cooling system for single to multiple detector systems [7739-154]

J. L. Lizon, M. Accardo, European Southern Observatory (Germany)

7739 3F Liquid nitrogen pre-cooling of large infrared instrument at ESO [7739-155]

J. L. Lizon, European Southern Observatory (Germany)

773936 A very accurate filter wheel for a large field IR imager [7739-156]

J. L. Lizon, European Southern Observatory (Germany)

$77393 \mathrm{H} \quad$ A hybrid liquid nitrogen system for the cooling of the ESO OmegaCAM detector [7739-157] J. L. Lizon, A. Silber, G. Jakob, European Southern Observatory (Germany)

$773931 \quad$ Advanced high-cooling power 2-stage Gifford-McMahon refrigerator systems [7739-158] G. Jakob, J. L. Lizon, European Southern Observatory (Germany)

7739 3J First concept for the E-ELT cryogenic infrastructure [7739-159] J. L. Lizon, J. C. Gonzalez, European Southern Observatory (Germany); C. Monroe, Monroe Brothers Ltd. (United Kingdom); I. Bryson, D. Montgomery, UK Astronomy Technology Ctr. (United Kingdom)

7739 3K An optical shutter for the Euclid imager [7739-161] A. M. Glauser, Institute for Astronomy, ETH Zurich (Switzerland) and UK Astronomy Technology Ctr. (United Kingdom); J. Amiaux, J.-L. Auguères, CEA, IRFU, SAp (France); S. Lilly, Institute for Astronomy, ETH Zurich (Switzerland); A. Refregier, CEA, IRFU, SAp (France)

$77393 \mathrm{~L} \quad$ Large format filter changer mechanism for the Dark Energy Survey [7739-162] G. Tarlé, B. Bigelow, D. Boprie, C. Cooper, E. Dede, W. Lorenzon, B. Nord, M. Schubnell, C. Weaverdyck, Univ. of Michigan (United States)

7739 3M Assembly of the Dark Energy Survey CCD Imager [7739-163]

G. Derylo, H. Cease, H. T. Diehl, J. Estrada, B. Flaugher, Fermi National Accelerator Lab. (United States)

7739 3N Cooling the Dark Energy Camera CCD array using a closed-loop two-phase liquid nitrogen system [7739-164]

H. Cease, Fermi National Accelerator Lab. (United States); D. DePoy, Texas A\&M Univ. (United States); G. Derylo, H. T. Diehl, J. Estrada, B. Flaugher, K. Kuk, Fermi National Accelerator Lab. (United States); S. Kuhlmann, Argonne National Lab. (United States); A. Lathrop, K. Schultz, R. J. Reinert, R. L. Schmitt, A. Stefanik, Fermi National Accelerator Lab. (United States); A. Zhao, Argonne National Lab. (United States)

773930 A precision lens mount for large temperature excursions [7739-166] S. A. Smee, The Johns Hopkins Univ. (United States) 
7739 3P The GRAVITY spectrometers: mechanical design [7739-169]

S. Fischer, M. Wiest, C. Straubmeier, S. Yazici, C. Araujo-Hauck, Univ. zu Köln (Germany); F. Eisenhaver, Max-Planck-Institut für extraterrestrische Physik (Germany); G. Perrin, Lab. d'Etudes Spatiales et d'Instrumetation en Astrophysique (France), Institut National des Sciences de I'Univers (France), and Groupement d'Intérêt Scientifique PHASE (France); W. Brandner, Max-Planck-Institut für Astronomie (Germany); K. Perraut, Lab. d'Astrophysique de l'Observatoire de Grenoble (France); A. Amorim, Univ. de Lisboa (Portugal); M. Schöller, European Southern Observatory (Germany); A. Eckart, Univ. zu Köln (Germany) and Max-Planck-Institut für Radioastroniomie (Germany)

$77393 \mathrm{Q}$ Prototyping and testing of mechanical components for the GRAVITY spectrometers [7739-170]

M. Wiest, S. Fischer, Univ. zu Köln (Germany); M. Thiel, M. Haug, Max-Planck-Institut für extraterrestrische Physik (Germany); R-R. Rohloff, Max-Planck-Institut für Astronomie (Germany); C. Straubmeier, C. Araujo-Hauck, S. Yazici, Univ. zu Köln (Germany); F. Eisenhaver, Max-Planck-Institut für extraterrestrische Physik (Germany); G. Perrin, Lab. d'Etudes Spatiales et d'Instrumentation en Astrophysique (France) and Institut National des Sciences de l'Univers (France); W. Brandner, Max-Planck-Institut für Astronomie (Germany); K. Perraut, Lab. d'Astrophysique Observatoire de Grenoble (France); A. Amorim, Univ. de Lisboa (Portugal); M. Schöller, European Southern Observatory (Germany); A. Eckart, Univ. zu Köln (Germany) and Max-Planck-Institut für Radioastronomie (Germany)

$77393 R \quad$ HARPS secondary guiding [7739-171]

G. Ihle, G. Avila, I. Kastinen, G. Lo Curto, A. Segovia, P. Sinclaire, European Southern Observatory (Germany); R. Tomelleri, Tomelleri s.r.l. (Italy)

773935 High-performance silicon grisms for $1.2-8.0 \mu \mathrm{m}$ : detailed results from the JWST-NIRCam devices [7739-173]

M. Gully-Santiago, W. Wang, C. Deen, The Univ. of Texas at Austin (United States); D. Kelly, The Univ. of Arizona (United States); T. P. Greene, NASA Ames Research Ctr. (United States); J. Bacon, II-VI Inc. (United States); D. T. Jaffe, The Univ. of Texas at Austin (United States)

7739 3т Optomechanical system of AlT tools to perform tests and integrations of 24 IFU [7739-174] E. Renault, F. Laurent, L. Adjali, R. M. Bacon, D. Boudon, P. Caillier, E. Daguisé, J.-P. Dubois, Univ. de Lyon (France), Observatoire de Lyon (France), CNRS (France), and Ctr. de Recherche Astrophysique de Lyon (France); H. Anwand, Georg-August-Univ. (Germany); J. Kosmalski, M. Loupias, Univ. de Lyon (France), Observatoire de Lyon (France), CNRS (France), and Ctr. de Recherche Astrophysique de Lyon (France); H. E. Nicklas, Georg-August-Univ. (Germany); A. Remillieux, Univ. de Lyon (France), Observatoire de Lyon (France), CNRS (France), and Ctr. de Recherche Astrophysique de Lyon (France)

$77393 \mathrm{U}$ Progress in the fabrication of a prototype ZnSe immersion grating for the WINERED spectrograph [7739-175]

P. J. Kuzmenko, S. L. Little, Lawrence Livermore National Lab. (United States); Y. Ikeda, Photocoding Inc. (Japan); N. Kobayashi, Institute of Astronomy, The Univ. of Tokyo (Japan) 
7739 3W ESPRESSO: design and analysis of Coudé-Train concepts for stable and efficient optical feeding [7739-178]

A. Cabral, A. Moitinho, J. Coelho, J. Lima, P. Carvas, A. Amorim, J. Rebordão, Univ. de Lisboa (Portugal); G. Ávila, European Southern Observatory (Germany); D. Mégevand, Observatoire de l'Univ. de Genève (Switzerland); J-M. Herreros, Instituto de Astrofísica de Canarias (Spain); F. Zerbi, INAF, Osservatorio Astronomico di Brera (Italy); P. Di Marcantonio, INAF, Osservatorio Astronomico di Trieste (Italy); C. Lovis, Observatoire de l'Univ. de Genève (Switzerland); N. C. Santos, Univ. do Porto (Portugal); F. Pepe, Observatoire de l'Univ. de Genève (Switzerland); S. Cristiani, INAF, Osservatorio Astronomico di Trieste (Italy); R. Rebolo, Instituto de Astrofísica de Canarias (Spain)

7739 3X Scattered light in a DMD based multi-object spectrometer [7739-195]

K. D. Fourspring, Z. Ninkov, J. P. Kerekes, Rochester Institute of Technology (United States)

POSTER SESSION: COATINGS/FILTERS/CLEANING/MASKS

7739 3Y Progress toward high-performance astronomical coatings [7739-180]

A. C. Phillips, W. E. Brown, B. Dupraw, D. F. Hilyard, D. J. Cowley, Univ. of California Observatories (United States)

773932 Cleaning the Southern African Large Telescope's M5 mirror [7739-182]

L. A. Crause, H. Gajjar, J. Love, F. Strümpfer, J. E. O'Connor, D. E. O'Donoghue, O. J. Strydom, D. A. H. Buckley, P. Gillingham, South African Astronomical Observatory (South Africa)

773940 Blocking filters with enhanced throughput for $\mathbf{x}$-ray microcalorimetry [7739-184]

D. A. Grove, J. C. Betcher, B. Lairson, R. Smith, T. Ayers, Luxel Corp. (United States)

773942 Pass-band filter performance for space-flight Dark Energy missions [7739-186]

J. Edelstein, Univ. of California, Berkeley (United States); S. L. Mufson, Indiana Univ. (United

States); N. J. Mostek, Lawrence Berkeley National Lab. (United States); B. J. Baptista, Indiana Univ. (United States); B. E. Woodgate, NASA Goddard Space Flight Ctr. (United States); A. G. Kim, Lawrence Berkeley National Lab. (United States); C. R. Bower, Indiana Univ. (United States); R. Boucarut, M. A. Quijada, NASA Goddard Space Flight Ctr. (United States)

\section{POSTER SESSION: PHOTONICS/ADC/ADAPTIVE OPTICS}

773944 Development of five multifibre links for the OPTIMOS-EVE study for the E-ELT [7739-188] I. Guinouard, F. Chemla, H. Flores, J.-M. Huet, F. Hammer, Observatoire de Paris (France); G. Wulterkens, Radboud Univ. Nijmegen (Netherlands)

773946 Multi-way optical fibre connectors for astronomy [7739-190]

D. M. Haynes, R. Haynes, W. Rambold, Anglo-Australian Observatory (Australia) and Astrophysikalisches Institut Potsdam (Germany); M. Goodwin, E. J. Penny, Anglo-Australian Observatory (Australia) 
773947 New scramblers for precision radial velocity: square and octagonal fibers [7739-191]

B. Chazelas, F. Pepe, F. Wildi, Observatory of Geneva, Univ. of Geneva (Switzerland);

F. Bouchy, Institut d'Astrophysique de Paris, CNRS, Univ. Pierre \& Marie Curie (France) and Observatoire de Haute-Provence, CNRS/OAMP, St. Michel l'Observatoire (France); S. Perruchot, Observatoire de Haute-Provence, CNRS/OAMP, St. Michel l'Observatoire (France); G. Avila, European Southern Observatory (Germany)

773948 The ADC for the VST Telescope: theory and preliminary test of the electromechanical system [7739-193]

P. Schipani, INAF, Osservatorio Astronomico di Capodimonte (Italy); J. Farinato, INAF, Osservatorio Astronomico di Padova (Italy); C. Arcidiacono, INAF, Osservatorio Astrofisico di Arcetri (Italy); S. D'Orsi, L. Ferragina, D. Fierro, INAF, VSTCeN (Italy); D. Magrin, INAF, Osservatorio Astronomico di Padova (Italy); L. Marty, INAF, VSTCeN (Italy); F. Perrotta, INAF, Osservatorio Astronomico di Capodimonte (Italy); R. Ragazzoni, INAF, Osservatorio Astronomico di Padova (Italy); G. Umbriaco, INAF, Univ. degli Studi di Padova (Italy)

\section{SESSION 12 CRYOGENIC INSTRUMENTS}

773949 Cryogenic submicron linear actuator (Invited Paper) [7739-82]

J. Serrano, J. Moreno Raso, D. González de María, H. Argelaguet Vilaseca, M. Lamensans,

D. López Justo, V. Sanz Puig, LIDAX (Spain)

7739 4A Design and prototype performance of an innovative cryogenic tip-tilt mirror [7739-83]

H. Janssen, M. Teuwen, Janssen Precision Engineering B.V. (Netherlands); R. Navarro,

N. Tromp, E. Elswijk, H. Hanenburg, NOVA-ASTRON (Netherlands)

7739 4B Different ways of reducing vibrations induced by cryogenic instruments [7739-84] J. L. Lizon, G. Jakob, European Southern Observatory (Germany); B. de Marneffe, A. Preumont, Univ. Libre de Bruxelles (Belgium)

7739 4C Ultrastable operation of detectors for high-resolution spectrographs [7739-85] A. Manescau, European Southern Observatory (Germany); M. Gullieuszik, Royal Observatory of Belgium (Belgium); O. Iwert, H. Dekker, J.-L. Lizon, G. Lo Curto, European Southern Observatory (Germany); P. Sinclaire, European Southern Observatory (Chile); F. M. Zerbi, INAF, Osservatorio di Brera (Italy); G. Jakob, European Southern Observatory (Germany); D. Sosa, Instituto de Astrofisica de Canarias (Spain); P. Amico, L. Pasquini, European Southern Observatory (Germany)

7739 4D Cryogenic Fourier transform infrared spectrometer from 4 to 20 micrometers [7739-86] S. G. Kaplan, S. I. Woods, T. M. Jung, A. C. Carter, National Institute of Standards and Technology (United States)

7739 4E A novel athermal approach for high-performance cryogenic metal optics [7739-87] R.-R. Rohloff, Max-Planck-Institut für Astronomie (Germany); A. Gebhardt, Fraunhofer-Institut für Angewandte Optik und Feinmechanik (Germany); V. Schönherr, Max-Planck-Institut für Astronomie (Germany); S. Risse, J. Kinast, S. Scheiding, T. Peschel, Fraunhofer-Institut für Angewandte Optik und Feinmechanik (Germany) 
7739 4G Fabrication and current optical performance of a large diamond-machined ZnSe immersion grating [7739-90]

Y. Ikeda, Photocoding (Japan) and Kyoto-Sangyo Univ. (Japan); N. Kobayashi, The Univ. of Tokyo (Japan); P. J. Kuzmenko, S. L. Little, Lawrence Livermore National Lab. (United States); C. Yasui, National Astronomical Observatory of Japan (Japan); S. Kondo, Kyoto-Sangyo Univ. (Japan); H. Mito, Kiso Observatory, The Univ. of Tokyo (Japan); K. Nakanishi, Kyoto-Sangyo Univ. (Japan); Y. Sarugaku, Japan Aerospace Exploration Agency (Japan)

$77394 \mathrm{H}$ Miniature spectrographs: characterization of arrayed waveguide gratings for astronomy [7739-91]

N. Cvetojevic, N. Jovanovic, Macquarie Univ. (Australia) and Australian Astronomical Observatory (Australia); J. Bland-Hawthorn, Sydney Institute for Astronomy, The Univ. of Sydney (Australia); R. Haynes, innoFSPEC, Astrophysikalisches Institut Potsdam (Germany); J. Lawrence, Macquarie Univ. (Australia) and Australian Astronomical Observatory (Australia)

773941 Miniature astronomical spectrographs using arrayed-waveguide gratings: capabilities and limitations [7739-92]

J. Lawrence, Macquarie Univ. (Australia) and Australian Astronomical Observatory (Australia); J. Bland-Hawthorn, Sydney Institute for Astronomy, The Univ. of Sydney (Australia); N. Cvetojevic, Macquarie Univ. (Australia); R. Haynes, innoFSPEC, Astrophysikalisches Institut Potsdam (Australia); N. Jovanovic, Macquarie Univ. (Australia) and Australian Astronomical Observatory (Australia)

\section{SESSION 14 SPECTROSCOPY/SLICERS II}

$77394 \mathrm{~J}$ Fibre optical spectroscopy and sensing innovation at innoFSPEC Potsdam [7739-93] R. Haynes, innoFSPEC Potsdam, Astrophysikalisches Institut Potsdam (Germany); O. Reich, innoFSPEC Potsdam, Univ. Potsdam (Germany); W. Rambold, innoFSPEC Potsdam, Astrophysikalisches Institut Potsdam (Germany); R. Hass, innoFSPEC Potsdam, Univ. Potsdam (Germany); K. Janssen, innoFSPEC Potsdam, Astrophysikalisches Institut Potsdam (Germany) and innoFSPEC Potsdam, Univ. Potsdam (Germany)

$77394 \mathrm{~L} \quad$ Manufacturing of silicon immersion gratings for infrared spectrometers [7739-172] W. Wang, M. Gully-Santiago, C. Deen, The Univ. of Texas at Austin (United States); D. J. Mar, Liquidia Technologies, Inc. (United States); D. T. Jaffe, The Univ. of Texas at Austin (United States)

7739 4M MUSE integral field unit: test results on the first out of 24 [7739-95]

F. Laurent, L. Adjali, Univ. de Lyon (France), Observatoire de Lyon (France), and Ctr. de Recherche Astrophysique de Lyon, CNRS, Ecole Normale Supérieure de Lyon (France); J. Arns, Kaiser Optical Systems, Inc. (United States); R. Bacon, D. Boudon, P. Caillier, E. Daguisé, Univ. de Lyon (France), Observatoire de Lyon (France), and Ctr. de Recherche Astrophysique de Lyon, CNRS, Ecole Normale Supérieure de Lyon (France); B. Delabre, European Southern Observatory (Germany); J. Dubois, Univ. de Lyon (France), Observatoire de Lyon (France), and Ctr. de Recherche Astrophysique de Lyon, CNRS, Ecole Normale Supérieure de Lyon (France); P. Godefroy, Winlight Optics \& Winlight System (France); A. Jarno, Univ. de Lyon (France), Observatoire de Lyon (France), and Ctr. de Recherche Astrophysique de Lyon, CNRS, Ecole Normale Supérieure de Lyon (France); P. Jorden, E2V 
technologies plc (United Kingdom); J. Kosmalski, Univ. de Lyon (France) and Observatoire de Lyon (France) and Ctr. de Recherche Astrophysique de Lyon, CNRS, Ecole Normale Supérieure de Lyon (France); V. Lapère, Winlight Optics \& Winlight System (France); J. Lizon, European Southern Observatory (Germany); M. Loupias, A. Pecontal, Univ. de Lyon (France) and Observatoire de Lyon (France) and Ctr. de Recherche Astrophysique de Lyon, CNRS, Ecole Normale Supérieure de Lyon (France); R. Reiss, European Southern Observatory (Germany); A. Remillieux, E. Renault, Univ. de Lyon (France) and Observatoire de Lyon (France) and Ctr. de Recherche Astrophysique de Lyon, CNRS, Ecole Normale Supérieure de Lyon (France); G. Rupprecht, European Southern Observatory (Germany); Y. Salaun, Winlight Optics \& Winlight System (France)

7739 4N ERASMUS-F: pathfinder for an E-ELT 3D instrumentation (Invited Paper) [7739-96] A. Kelz, M. M. Roth, Astrophysikalisches Institut Potsdam (Germany); R. Bacon, Ctr. de Recherche Astronomique de Lyon (France); J. Bland-Hawthorn, Sydney Institute for Astronomy, The Univ. of Sydney (Australia); H. E. Nicklas, Institut für Astrophysik Göttingen, Georg-August-Univ. Göttingen (Germany); J. J. Bryant, Sydney Institute for Astronomy, The Univ. of Sydney (Australia); M. Colless, Australian Astronomical Observatory (Australia); S. Croom, S. Ellis, Sydney Institute for Astronomy, The Univ. of Sydney (Australia); A. Fleischmann, Institut für Astrophysik Göttingen, Georg-August-Univ. Göttingen (Germany); P. Gillingham, Australian Astronomical Observatory (Australia); R. Haynes, innoFSPEC Potsdam (Germany) and Australian Astronomical Observatory (Australia); A. Hopkins, Australian Astronomical Observatory (Australia); J. Kosmalski, Ctr. de Recherche Astronomique de Lyon (France); J. W. O'Byrne, Sydney Institute for Astronomy, The Univ. of Sydney (Australia); J-C. Olaya, W. N. Rambold, innoFSPEC Potsdam (Germany); G. Robertson, Sydney Institute for Astronomy, The Univ. of Sydney (Australia)

773940 Volume phase holographic echelle grating: a theoretical study [7739-97] A. Bianco, G. Pariani, INAF, Osservatorio Astronomico di Brera (Italy) and Politecnico di Milano (Italy)

$77394 \mathrm{P}$ Photochromic polymers for making volume phase holographic gratings: between theory and practice [7739-98]

A. Bianco, INAF, Osservatorio Astronomico di Brera (Italy); G. Pariani, INAF, Osservatorio Astronomico di Brera (Italy) and Politecnico di Milano (Italy); C. Bertarelli, Politecnico di Milano (Italy) and Ctr. for Nano Science and Technology (Italy); F. M. Zerbi, INAF, Osservatorio Astronomico di Brera (Italy)

$77394 \mathrm{Q}$ Development of MEMS-based programmable slit mask for multi-object spectroscopy [7739-99]

M. Canonica, Ecole Polytechnique Fédérale de Lausanne (Switzerland); S. Waldis, Univ. de Neuchâtel (Switzerland); F. Zamkotsian, P. Lanzoni, Lab. d'Astrophysique de Marseille (France); W. Noell, N. De Rooij, Ecole Polytechnique Fédérale de Lausanne (Switzerland)

7739 4R Efficiency measurements performed on the MUSE VPHG [7739-100]

E. Renault, M. Loupias, L. Adjali, Univ. de Lyon (France), Observatoire de Lyon (France), and Ctr. de Recherche Astrophysique de Lyon, CNRS, Ecole Normale Supérieure de Lyon (France); J. A. Arns, Kaiser Optical Systems, Inc. (United States); R. Bacon, D. Boudon, P. Caillier, P. Coadour, Univ. de Lyon (France), Observatoire de Lyon (France), and Ctr. de Recherche Astrophysique de Lyon, CNRS, Ecole Normale Supérieure de Lyon (France); H. Dekker, European Southern Observatory (Germany); J.-P. Dubois, J. Kosmalski, Univ. de Lyon (France), Observatoire de Lyon (France), and Ctr. de Recherche Astrophysique de 
Lyon, CNRS, Ecole Normale Supérieure de Lyon (France); L. Pinard, Lab. des Matériaux Avancés, CNRS (France); A. Remillieux, Univ. de Lyon (France), Observatoire de Lyon (France), and Ctr. de Recherche Astrophysique de Lyon, CNRS, Ecole Normale Supérieure de Lyon (France)

7739 4S The SOAR integral field unit spectrograph optical design and IFU implementation [7739-101] A. C. de Oliveira, L. S. de Oliveira, C. D. Gneiding, Lab. Nacional de Astrofísica (Brazil); B. Barbuy, Instituto Astronômico e Geofísico, Univ. de São Paulo (Brazil); D. Jones, Prime Optics (Australia); M. V. Figueredo, Univ. Federal do Vale do São Francisco (Brazil); J. R. D. Lépine, Instituto Astronômico e Geofísico, Univ. de São Paulo (Brazil);

V. B. P. Macanhan, J. B. C. de Oliveira, Lab. Nacional de Astrofísica (Brazil); K. Taylor, Instituto Astronômico e Geofísico, Univ. de São Paulo (Brazil)

$77394 \mathrm{~T} \quad$ Combining laser frequency combs and iodine cell calibration techniques for Doppler detection of exoplanets [7739-102]

K. Cahoy, NASA Ames Research Ctr. (United States); D. Fischer, J. Spronck, D. DeMille, Yale Univ. (United States)

$77394 \mathrm{U}$ The Brazilian tunable filter imager for SOAR [7739-103]

K. Taylor, C. Mendes de Oliveira, Univ. de São Paulo (Brazil); R. Laporte, Instituto Nacional de Pesquisas Espaciais (Brazil); C. D. Guzman, Astrolnventions (Chile); J. Ramirez Fernandez, Politécnica da Univ. de São Paulo (Brazil); S. Scarano, Jr., G. Ramos, Univ. de São Paulo (Brazil); H. Plana, Univ. Estadual de Santa Cruz (Brazil); F. E. Lourenco, Univ. Paulista (Brazil); J.-L. Gach, Lab. d'Astrophysique de Marseille (France); F. L. Fontes, XNOVA (Brazil); F. Ferrari, Univ. Federal do Pampa (Brazil); L. Cavalcanti, E. C. Gutierrez Castañeda, A. de Calasans, Univ. de São Paulo (Brazil); P. Balard, P. Amram, Univ. Estadual de Santa Cruz (Brazil);

D. Andrade, Univ. de São Paulo (Brazil)

Author Index 


\title{
Conference Committee
}

\author{
Symposium Chairs
}

Masanori lye, National Astronomical Observatory of Japan (Japan)

Douglas A. Simons, Gemini Observatory (United States)

Symposium Cochairs

Mark M. Casali, European Organisation for Astronomical Research in the Southern Hemisphere (Germany)

Kathryn A. Flanagan, Space Telescope Science Institute (United States)

Conference Chairs

Eli Atad-Ettedgui, UK Astronomy Technology Center/The Royal Observatory Edinburgh (United Kingdom)

Dietrich Lemke, Max-Planck-Institut für Astronomie (Germany)

Program Committee

Joseph Antebi, Simpson Gumpertz \& Heger Inc. (United States)

Daniel R. Blanco, National Optical Astronomy Observatory (United States)

Colin R. Cunningham, UK Astronomy Technology Center (United Kingdom)

V. Alfonso Feria, Jet Propulsion Laboratory (United States)

Roland Geyl, Sagem SA (France)

Peter Hartmann, SCHOTT AG (Germany)

David Montgomery, UK Astronomy Technology Center (United Kingdom)

Eric Prieto, Observatoire Astronomique de Marseille-Provence (France)

Session Chairs

1 Optics Fabrication I

Eli Atad-Ettedgui, UK Astronomy Technology Center/The Royal Observatory Edinburgh (United Kingdom)

2 Optics Fabrication II

Eli Atad-Ettedgui, UK Astronomy Technology Center/The Royal Observatory Edinburgh (United Kingdom)

3 Materials/Metrology

Peter Hartmann, SCHOTT AG (Germany) 
4 Test and Metrology I

Peter Hartmann, SCHOTT AG (Germany)

5 Test and Metrology II

Eric Prieto, Observatoire Astronomique de Marseille-Provence (France)

6 Test and Metrology III

Eli Atad-Ettedgui, UK Astronomy Technology Center/The Royal Observatory Edinburgh (United Kingdom)

$7 \quad$ Cryogenic Space Instruments

Dietrich Lemke, Max-Planck-Institut für Astronomie (Germany)

8 Telescope Structure I

Joseph Antebi, Simpson Gumpertz \& Heger Inc. (United States)

9 Telescope Structure II

V. Alfonso Feria, Jet Propulsion Laboratory (United States)

10 Coatings

Eric Prieto, Observatoire Astronomique de Marseille-Provence (France)

11 Photonics

Colin R. Cunningham, UK Astronomy Technology Center (United Kingdom)

12 Cryogenic Instruments

Dietrich Lemke, Max-Planck-Institut für Astronomie (Germany)

13 Spectroscopy/Slicers I

David Montgomery, UK Astronomy Technology Center (United Kingdom)

14 Spectroscopy/Slicers II

Eli Atad-Ettedgui, UK Astronomy Technology Center/The Royal Observatory Edinburgh (United Kingdom) 


\section{Introduction}

Astronomy is driven by the desire to understand our position in the universe, and by new technology.

For almost forty years, SPIE has organized conferences on astronomical telescopes and instrumentation. These events eventually became huge symposia with specialized parallel conferences on all major instrumental areas, such as space- and ground-based telescopes, detectors, interferometers, and adaptive optics. With the increasing complexity of the instrumentation; however, a further conference dedicated to the enabling technologies became necessary. This conference "Modern Technologies in Space- and Ground-based Telescopes and Instrumentation" was held for the first time in 2002 as part of the SPIE symposium of several conferences on astronomical telescopes and instrumentation, attended by $\sim 2000$ scientists and technicians. While the first two of these new technologyconferences started off with 47 and 79 contributions, respectively, the number of papers has increased to $\sim 200$ at this fifth conference. Six full days were necessary to schedule about half of the submitted contributions for oral presentations; the rest were posters. Several participants felt somewhat exhausted after a full conference week, which started early on a Sunday morning. The readers of these proceedings are in a more comfortable position: they can take their time to study the interesting and well written contributions from scientists and technicians from laboratories all over the world. However, they will of course miss out on the pleasure of listening to the number of committed speakers, and they cannot ask questions in real time.

This volume documents the impressive progress made in the technical preparation for the extremely large telescopes on both sides of the Atlantic since the 2008 meeting. The first large mirror segment for the Giant Magellan Telescope GMT was presented, including the alignment and test strategy for this $8 \mathrm{~m}$-off-axis giant. A first series of the $1.4 \mathrm{~m}$-hexagon segments for the $42 \mathrm{~m}$ European counterpart E-ELT, made of different materials, proved that ESO and the European industry are ready to start building this giant telescope. Even "old" mirror materials such as Zerodur are good for a surprise. Its mechanical strength could recently be improved and measured to be nearly an order of magnitude higher than listed so far. This enables the manufacturing of "superlightweight" glass ceramic mirrors of only $\sim 15 \%$ the mass of a full blank.

"Astrophotonics" was another major topic of this conference. With its novel devices such as starbugs, miniature spectrographs in multi-objects spectroscopy, array waveguide gratings, fiber Bragg and VPH gratings, it will revolutionize astronomy and reduce the size, mass, and cost of the next generation of instruments. 
But sometimes progress at one front means a disaster at another: The modern closed cycle coolers for the large infrared instruments at the Very Large Telescope VLT have displayed a vibration level which is unacceptable for the operation of interferometers at the VLT- I. The solutions of the "vibration war" presented here look like an apparent, but sophisticated step backwards to Linde's good old liquid nitrogen.

We hope that the readers will find reading these proceedings exciting, and that they will stimulate ideas for their own research.

We look forward to seeing you at the next conference on "Modern Technologies..." in Amsterdam in the summer of 2012.

Eli Atad-Ettedgui Dietrich Lemke 